\title{
In silico approach to investigating the adsorption mechanisms of short chain perfluorinated sulfonic acids and perfluorooctane sulfonic acid on hydrated hematite surface
}

\author{
Hongru Feng a, b, Yuan Lin ${ }^{a}$, Yuzhen Sun ${ }^{a}$, Huiming Cao ${ }^{a}$, Jianjie Fu ${ }^{\text {a }}$, Ke Gao ${ }^{\text {a }}$, \\ Aiqian Zhang ${ }^{\text {a, }}$, * * \\ a State Key Laboratory of Environmental Chemistry and Ecotoxicology, Research Center for Eco-Environmental Sciences, Chinese Academy of Sciences, \\ Beijing 100085, China \\ ${ }^{\mathrm{b}}$ College of Resources and Environment, University of Chinese Academy of Sciences, Beijing 100190, China
}

\section{A R T I C L E I N F O}

\section{Article history:}

Received 4 September 2016

Received in revised form

1 February 2017

Accepted 11 February 2017

Available online 14 February 2017

\section{Keywords:}

Perfluorinated sulfonic acids

Forcite

CASTEP

Adsorption

\begin{abstract}
A B S T R A C T
Short chain perfluorinated sulfonic acids (PFSAs) that were introduced as alternatives for perfluorooctane sulfonic acid (PFOS) have been widely produced and used. However, few studies have investigated the environmental process of short chain PFSAs, and the related adsorption mechanisms still need to be uncovered. The water-oxide interface is one of the major environmental interfaces that plays an important role in affecting the adsorption behaviour and transport potential of the environmental pollutant. In this study, we performed molecular dynamics simulations and quantum chemistry calculations to investigate the adsorption mechanisms of five PFSAs and their adsorption on hydrated hematite surface as well. Different to the vertical configuration reported for PFOS on titanium oxide, all PFSAs share the same adsorption configuration as the long carbon chains parallel to the surface. The formation of hydrogen bonds between $\mathrm{F}$ and inter-surface $\mathrm{H}$ helps to stabilize the unique configuration. As a result, the sorption capacity increases with increasing C-F chain length. Moreover, both calculated adsorption energy and partial density of states (PDOS) analysis demonstrate a PFSAs adsorption mechanism in between physical and chemical adsorption because the hydrogen bonds formed by the overlap of $\mathrm{F}(p)$ orbital and $\mathrm{H}(s)$ orbital are weak intermolecular interactions while the physical adsorption are mainly ascribed to the electrostatic interactions. This massive calculation provides a new insight into the pollutant adsorption behaviour, and in particular, may help to evaluate the environmental influence of pollutants.
\end{abstract}

(c) 2017 Published by Elsevier Ltd.

\section{Introduction}

Perfluoroalkyl acids are widely used as surfactants in various industrial and consumer products such as lubricants, paints, metal cleaners, cosmetics, and fire-fighting foams (Matyszewska et al.,

\footnotetext{
Abbreviations: PFSA, perfluorinated sulfonic acids; PFBS, perfluorobutane sulfonic acid; PFPS, perfluoropentane sulfonic acid; PFHxS, perfluorohexane sulfonic acid; PFHpS, perfluoroheptane sulfonic acid; PFOS, perfluorooctane sulfonic acid; DFT, density functional theory; MD, molecular dynamics; COMPASS, condensedphase optimized molecular potentials for atomistic simulation studies; RDF, radial distribution function.

* Corresponding author. State Key Laboratory of Environmental Chemistry and Ecotoxicology, Research Center for Eco-Environmental Sciences, Chinese Academy of Sciences, Beijing 100085, China.
}

E-mail address: aqzhang@rcees.ac.cn (A. Zhang).
2007). They have been detected in the environment and drinking water and have been found to be present in humans and a wide range of biota (Giesy and Kannan, 2001; Kannan et al., 2004; Rahman et al., 2014; Thomas, 2006). Due to their persistence, toxicity, bioaccumulative behaviour, and the capacity for longrange transport, they have attracted worldwide attention; this is especially true for perfluorooctane sulfonic acid (PFOS) and perfluorooctane sulfonyl fluoride (POSF)-based chemicals that were listed under Annex B (restriction of production and use) of the UNEP Stockholm Convention in 2009 (UNEP, 2009). Hence, the short chain perfluorinated sulfonic acids (PFSAs, $\mathrm{C}_{n} \mathrm{~F}_{2 \mathrm{n}+1} \mathrm{SO}_{3} \mathrm{H}$ ) with fewer than seven perfluorinated carbons and their salts were produced and used as alternative compounds due to their identical functional properties and lower bioaccumulation (Olsen et al., 2009; Wang et al., 2013). Previous studies have reported that 
PFBS was found to be the dominant PFAAs in the Rhine River watershed, and drinking water in Germany and the Tangxun Lake (Lange et al., 2007; Moller et al., 2010; Zhou et al., 2013). Therefore, attention should be concentrated on the environmentally related processes of the PFSAs in aquatic environment. Despite the increasing production of short chain PFSAs and the fact that short chain PFSAs have spread worldwide, little is known about their processes in the environment.

Due to the heterogeneity in the environmental interface, aqueous and mineral surface chemical behaviours are the major controlling factors in the fate and transport of organic pollutants. Mineral surfaces that are often used as catalysts and adsorbents play a key role in several environmental processes. Previous studies have shown that mineral surfaces in contact with ground water can adsorb and catalyze reactions of pollutant molecules (Al-Abadleh and Grassian, 2003). Hematite $\left(\alpha-\mathrm{Fe}_{2} \mathrm{O}_{3}\right)$ is a common and ubiquitous environmental sorbent and thus plays an important role in contaminant sequestration and chemical speciation in groundwater aquifers and soils (Jr et al., 1999b), which in turn affect the toxicity and potential bioavailability of contaminants ( $\mathrm{Jr}$ et al., 1999a). Studies of environmental processes provide the basis for revealing molecular-level information about adsorbate-surface interactions, interface reaction mechanisms, structure-reactivity relationships and an overall understanding of these processes at the molecular level.

To better reveal the mechanisms of the complex environmental process, advanced research techniques and methods are required. Scanning Tunneling Microscope (STM) (Eggleston et al., 2003), Fourier Transform Infrared Spectroscopy (FTIR) (Gao and Chorover, 2012) and X-ray scattering (Tanwar et al., 2007) methods have been used to reveal the images of surface structure and atomic arrangement in ambient conditions. In addition to these experimental techniques and methods, recently, there has been a rapid increase in publications of computational studies that can provide sufficiently detailed characterization of interface systems at the atomic level. Notman and Walsh (2009) investigated the interactions of water and the surface of $\alpha$-quartz by molecular dynamics simulations. Li et al. (2012) studied the glycine molecules in the gas phase on rutile $\mathrm{TiO}_{2}\left(\begin{array}{lll}1 & 1 & 0\end{array}\right)$ surface in a water solution by standard classical molecular dynamics simulations and reactive dynamics simulations.

In this study, we used density functional theory (DFT) and molecular dynamics (MD) simulations to investigate the adsorption of PFSAs in environment. As in the construction of the periodic structure of hematite surface, simple repeat unit and considerable computational effort are required to obtain reasonable results. We then constructed a hydroxylated surface in the structure similar to the $(\mathrm{HO})_{3}-\mathrm{Fe}-\mathrm{H}_{3} \mathrm{O}_{3}-\mathrm{R}$ structure (Trainor et al., 2004) and relaxed it using the DFT method. In the construction of a system containing water molecules and hematite surface for performing MD simulations, it is clear that water is likely ordered to some extent above the surfaces of hematite (Kerisit, 2011). Therefore, we left sufficient space to avoid the influence of two interfaces in the periodic structure. To the best of our knowledge, this is the first study to reveal the adsorption mechanics of different PFSAs in silico, and combination of two methods can effectively provide the necessary information on different perspectives to reveal the PFSAs behaviour and mechanisms on the hematite surface.

\section{Computational details and models}

\subsection{Computational details}

All simulations were carried out with the commercial Materials Studio software package (Accelrys Software Inc, 2012). The simulation methodology includes molecular dynamics calculations using the Forcite module and density functional theory calculations using the CASTEP module (Clark et al., 2005).

MD was performed in the periodic canonical ensemble (NVT) using the COMPASS force field, which is one of the first ab initio force field approaches that has been parameterized and validated using the gas-phase and condensed-phase properties of organic and inorganic materials (Sun, 1998; Sun et al., 1998). The minimization was performed using the Smart algorithm that automatically combines appropriate features of the other available methods in a cascade. Simulations were carried out in the NVT ensemble using the Nosé algorithm (Karasawa and Iii, 1989; Nosé, 1991, 2006, 1984) for a run time of $4 \mathrm{~ns}$ with a $1 \mathrm{fs}$ time step at $298 \mathrm{~K}$. The simulations were initialized with the minimum-energy structures and random initial velocities. The non-bonded interactions were calculated using the Ewald (Hohenberg and Kohn, 2010; Williams, 2007) method.

To obtain a more accurate hematite structure, DFT calculations were carried out with the CASTEP module using the plane wave pseudopotential method (PWP) within the DFT formalism (Kohn and Sham, 1965). For the model of hematite $\left(\alpha-\mathrm{Fe}_{2} \mathrm{O}_{3}\right)$, spin polarization should be considered. The detailed parameters of spin direction, spin state and DFT $+U$ are found in previous studies (Martin et al., 2009; Rollmann et al., 2004; Trainor et al., 2004). More computational details are presented in Support Information, Text S1.

\subsection{Computational models}

The geometry optimized $\alpha-\mathrm{Fe}_{2} \mathrm{O}_{3}$ hexagonal unit cell $\left(\mathrm{a}=\mathrm{b}=5.02892 \AA, \mathrm{c}=13.7956 \AA, \alpha=\beta=90^{\circ}, \gamma=120^{\circ}\right)$ was in good agreement with the experimental data $(\mathrm{a}=\mathrm{b}=5.038 \AA$, $\mathrm{c}=13.772 \AA$ (Blake et al., 1966); $\mathrm{a}=\mathrm{b}=5.035 \AA$, $\mathrm{c}=13.747 \AA$ (Finger and Hazen, 1980)). The relaxed crystal was used to cleave the hematite $\left(\begin{array}{llll}0 & 0 & 0 & 1\end{array}\right)$ surface that was frequently observed on the naturally grown crystals and that is thermodynamically stable (Wang et al., 1998). Previous studies indicated that $\alpha-\mathrm{Fe}_{2} \mathrm{O}_{3}\left(\begin{array}{llll}0 & 0 & 0 & 1\end{array}\right)$ is Fe-terminated and that the highly relaxed single-layer $\mathrm{Fe}$ terminated $\mathrm{Fe}-\mathrm{O}_{3}-\mathrm{Fe}-\mathrm{R}$ surface is the most stable configuration (Alvarez-RamıRez et al., 2004; Chambers and Yi, 1999; Trainor et al., 2004; Wang et al., 1998; Wasserman et al., 1997) because this surface is stoichiometric and non-polar.

The surface was known to hydroxylate at water vapour pressures greater than $10^{-4}$ Torr. It was believed that the surface was fully hydroxylated in aqueous conditions (Liu et al., 1998). We noticed that there several different hydrated hematite $\left(\begin{array}{llll}0 & 0 & 0 & 1\end{array}\right)$ surfaces are known (Mason et al., 2009; Souvi et al., 2013; Wasserman et al., 1997), and in the consideration of our model and the ambient environment, the surface model was built symmetrically so that there is no net surface dipole. The construction of hydrated hematite $\left(\begin{array}{llll}0 & 0 & 0 & 1\end{array}\right)$ surface is presented detailedly in Support Information, Text S2 and Fig. S1. After geometry optimization, we obtained a surface relaxed structure that was similar to the structure found in a previous study (Kerisit, 2011; Mason et al., 2009).

The next consideration is the models of the PFSA and hydrated hematite $\left(\begin{array}{llll}0 & 0 & 0 & 1\end{array}\right)$ surface in environment for MD simulation. The calculated pKa of PFOS is -3.27 , and $3 \mathrm{M}$ confirm that the substance is considered to be a strong acid (Brooke et al., 2004). The reported pKa values of PFBS and PFHxS are all 0.14 (Steinle-Darling and Reinhard, 2008), and a previous study has suggested that the pKa values for PFSAs are below 0.3 (Vierke et al., 2013). Under the ground water $\mathrm{pH}$, considering the Henderson-Hasselbalch equation: 
$\mathrm{pH}=\mathrm{pKa}+\log \left(\left[\mathrm{A}^{-}\right] /[\mathrm{HA}]\right)$

$\left[\mathrm{A}^{-}\right] /[\mathrm{HA}]=10^{\mathrm{pH}-\mathrm{pKa}}$

it is obvious that PFSAs in the water are dissociated. The dissociated state and the non-dissociated state will give rise to different interactions with water and the interface, leading to different results obtained by computational methods, and we used the ionic state to be a closer representation of the real environment conditions.

PFSAs in the environment (e.g., soil, sediment, water) are always surrounded by water molecules. Therefore, it is reasonable to assume that any "realistic" model should include water molecules. The water molecules will present a regular arrangement at the hematite/water interface, and the atomic-level structure of water at surface is an important factor for controlling the interfacial reactions (Kerisit, 2011). The degree of ordering decreases with increasing distance from the surfaces and the ordered water extends at least $1 \mathrm{~nm}$ into the bulk fluid on the $\left(\begin{array}{llll}0 & 0 & 0 & 1\end{array}\right)$ hematite surface (Catalano, 2011). The existence of PFSA ${ }^{-}$also influences the distribution of water (Support Information, Text S3 and Fig. S2). Based on the above, we replicated the $(1 \times 1)$ two-surface hydrated cell into a $(5 \times 5)$ supercell with a vacuum layer of $60 \AA$ filled by liquid with a density of $1.01 \mathrm{~g} / \mathrm{cm}^{3}$ that consists of water molecules, one PFSA ${ }^{-}$ion and a potassium ion. The potassium ion was added to equilibrate the charge. This resulted in a large cell of $25.1445 \times 25.1445 \times 77.6730 \AA^{3}$ that contained more than a thousand water molecules. An example of the PFOS-containing system is shown in Fig. 1.

Adsorption experiments were carried out to support the calculation results. The experimental methods are described in Support Information, Text S4.

\section{Results and discussion}

\subsection{Adsorption configuration}

We present the results for PFSA adsorption on the hydrated hematite $\left(\begin{array}{llll}0 & 0 & 0 & 1\end{array}\right)$ surface obtained by MD simulations. During the simulations, $\mathrm{PFSA}^{-}$ion appeared to first move randomly in the aqueous solution. The configurations and orientations of the PFOS molecules were not constant or regular, but in general, they did show some trends of motion in the $Z$ direction toward the surface. PFBS, PFPS, PFHxS, PFHpS and PFOS adsorbed after a simulation time of $1.7 \mathrm{~ns}, 2.38 \mathrm{~ns}, 3.76 \mathrm{~ns} 1.2 \mathrm{~ns}$ and $1.64 \mathrm{~ns}$, respectively.

Fig. 2 shows the snapshots of the adsorbed frames that most configurations of the PFSA ${ }^{-}$ions in dynamic simulations lying on the hydrated hematite $\left(\begin{array}{llll}0 & 0 & 0 & 1\end{array}\right)$ surface. The adsorption is not constant. We noted that in the MD simulation, all $\mathrm{PFSA}^{-}$were flexible, could lie, twist or float on the hematite surface and did not stay in a fixed location. We speculated that the fluorine in the PFSA tail (perfluoroalkyl chain) plays an important role in the system. The hematite surface is highly hydrated resulting in similar surface morphology in the entire surface region. Therefore, at $298 \mathrm{~K}, \mathrm{PFSA}^{-}$ has the opportunity to transform and move from between the nearby regions.

It is interesting to see that the $\mathrm{PFSA}^{-}$adsorption configurations are not similar to those of alkyl sulfonic acid (e.g., sodium dodecyl sulfate SDS) adsorption on the hematite surface. The adsorption conformation of SDS likely has a different geometry with the first sorbate layer orienting the hydrophobic tail toward the solution (Gao and Chorover, 2010). A previous study focusing on the adsorption of sodium laurate on hematite particles showed that the laurate "stands" on the surface with the head toward the hematite and tail toward the aqueous solution (Chernyshova et al., 2011). As shown in Fig. 2, in our simulations, most of the adsorbed PFSA

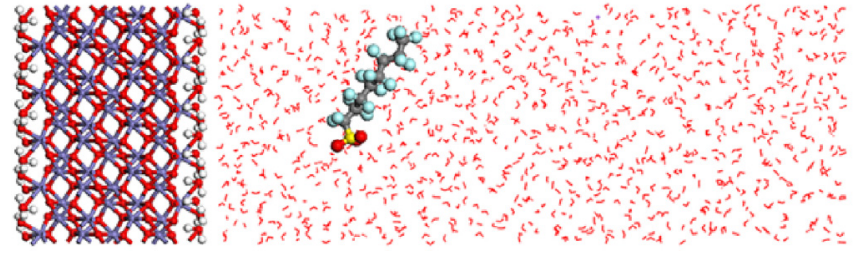

Fig. 1. Constructed model for MD simulation. Fluorine atoms are shown in cyan and carbon atoms in gray. (For interpretation of the references to colour in this figure legend, the reader is referred to the web version of this article.)

configurations are not "vertical". They interact with surface in the "horizontal" manner. The tail of both short chain PFSAs and PFOS is attached to the hydrated hematite surface with several hydrogen bonds of $F \cdots H$. This result is similar to the previous study that showed the adsorption isotherms for PFOS onto Ottawa sand, and demonstrated that the long axis is parallel to the surface on the basis of the molecule size and the level of coverage (Johnson et al., 2007). Zhao et al. (2014) concluded that PFOS has a longer carbon chain and could contact with more active sites of humic substances compared with PFHxS, indicating that the carbon chain plays an important role in adsorption. There were also a few configurations where the head group was oriented to the surface with a carbon chain bridge connected to the attached tail, similar to the " $\Omega$ "-shape (the configuration is not shown). Moreover, while a few trajectories indicated that the "stand" adsorption model did exist, it desorbed or changed configuration after a few picoseconds, demonstrating that the interaction between the PFSA head group and the surface is not sufficient to hold PFSA ${ }^{-}$to stand stably.

\subsection{Adsorption stability}

As a preliminary analysis of the adsorption models, we noticed that the PFSA adsorption structures on the hydrated hematite surface showed some unique styles. To verify whether the adsorption structures are stable, we performed another $1 \mathrm{~ns}$ dynamic simulations for each system. Fig. 3 shows the relative concentration of fluorine atoms during the dynamic simulations, where the top of the hydrated hematite $\left(\begin{array}{llll}0 & 0 & 0 & 1\end{array}\right)$ surface was set as zero point. Data were collected in the specified direction vertical to the surface. As shown in Fig. 3, there is a sharp peak of fluorine atom in $\mathrm{PFSA}^{-}$tail located around 1.8 Å from the surface, indicating that PFSA ${ }^{-}$adsorb onto the surface. And there is another peak
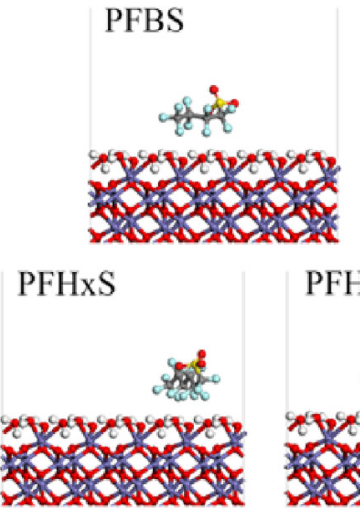

Fig. 2. Snapshots of the PFSA ${ }^{-}$being adsorbed on the hydrated hematite $\left(\begin{array}{llll}0 & 0 & 0 & 1\end{array}\right)$ surface. Fluorine atoms are shown in cyan, carbon atoms are shown in gray, oxygen atoms are shown in red, iron atoms are shown in violet and hydrogen atoms are shown in white. Water molecules are not shown. (For interpretation of the references to colour in this figure legend, the reader is referred to the web version of this article.) 


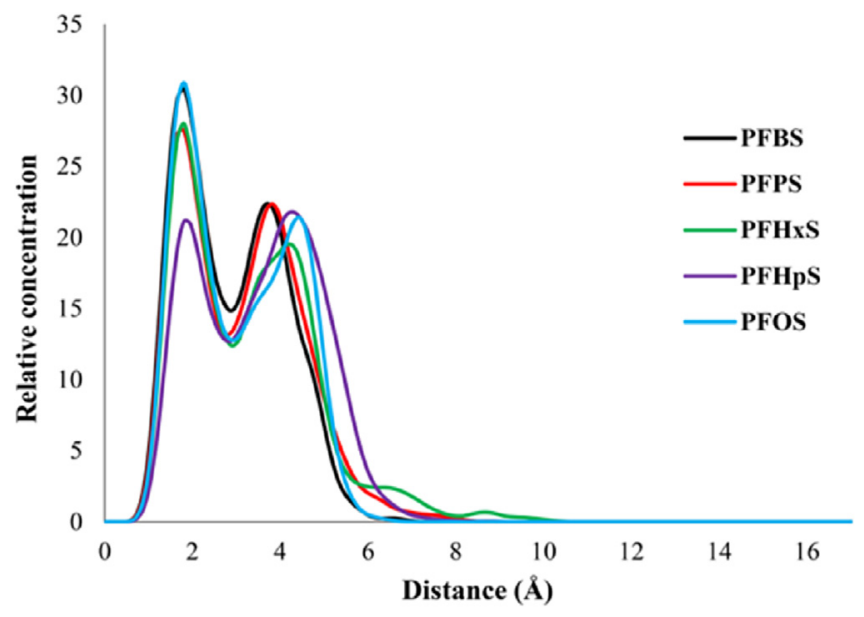

Fig. 3. Relative concentration of fluorine atoms as a function of distance from the hydrated hematite $\left(\begin{array}{llll}0 & 0 & 0 & 1\end{array}\right)$ surface.

around $4 \AA$, because of the PFSAs tails structure that have fluorine atoms on the other side. The fluorine atom cannot be detected at the distance above $10 \AA$. The relative concentration of fluorine atoms suggest that all the PFSAs in this study adsorb on the hydrated hematite surface strongly.

\subsection{Hydrogen bond}

As the relative concentration peak shown in Fig. 3, it is natural to consider the interaction between fluorine atoms and hydrated surface caused by hydrogen bonds (HB). Subsequently, we identify the hydrogen bond formed between the F atoms of $\mathrm{PFSA}^{-}$and the $\mathrm{O}$ atoms of the hydrated hematite surface using the following geometric criteria to determine whether an $\mathrm{HB}$ was formed: $\mathrm{d}_{\mathrm{F}} \ldots$ $\mathrm{o} \leq 3.5 \AA$ and $120^{\circ} \leq \theta_{\mathrm{O}-\mathrm{H} \cdots \mathrm{F}} \leq 180^{\circ}$ (Lu et al., 2010). The HBs from 400 stable adsorbed frames during 1 ns of the simulations were counted. Fig. 4 shows the distribution of the number of HBs for different length ranges of the $\mathrm{PFSA}^{-}$. These observed results clearly show a trend of increasing total number of HBs with increased carbon chain length. Generally, the values of the total number of HBs evaluated using different HB lengths also exhibit this tendency. For PFSA ${ }^{-}$, it is easy to figure out that because longer carbon chains

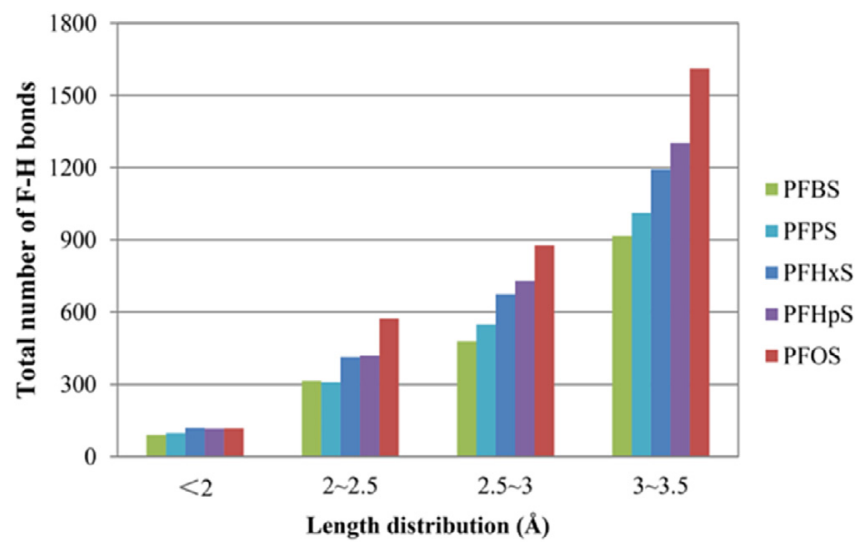

Fig. 4. Histogram of hydrogen bond distribution of $\mathrm{PFSA}^{-}$in different $\mathrm{d}_{\mathrm{F} \ldots \mathrm{o}}$ ranges. Horizontal axis denotes the different hydrogen bond lengths in angstrom. Vertical axis denotes the number of hydrogen bonds. Data for different PFSAs are shown in different colors. (For interpretation of the references to colour in this figure legend, the reader is referred to the web version of this article.)
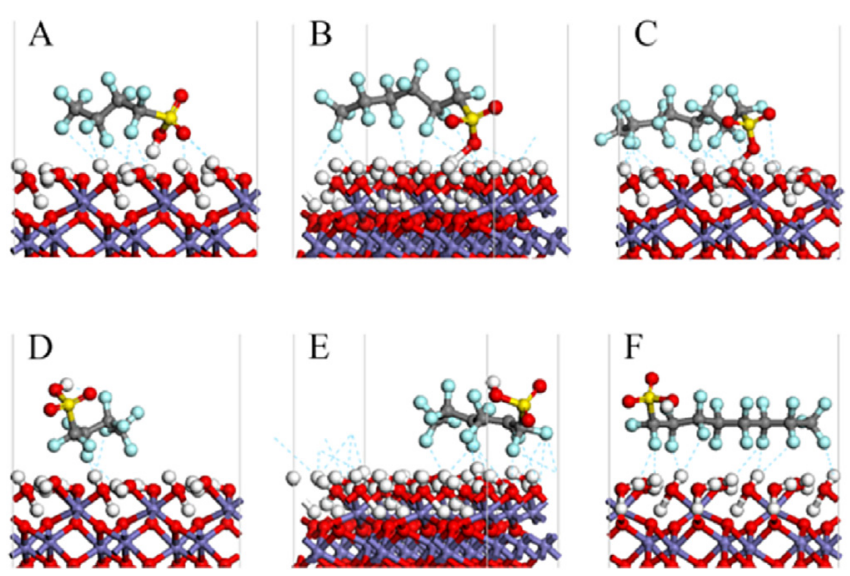

Fig. 5. Geometry optimized structures of PFSAs adsorbed on the hematite surface. A, B and $C$ show models of PFBS, PFHxS and PFOS adsorption on hematite surface with sulfonic group close to the surface. D, E and F show the PFBS, PFHxS and PFOS adsorption on hematite surface with sulfonic group away from surface.

have greater number of fluorine atoms, these chains have more opportunities to form HBs between the $\mathrm{F}$ atoms of $\mathrm{PFSA}^{-}$and the $\mathrm{O}$ atoms of the hydrated hematite surface. At the same time, the adsorption strength can be enhanced by increasing number of HBs. Moreover, the distribution of strong hydrogen bond (simply classified by the distance smaller than $2.5 \AA$ ) also makes a difference. Among the studied PFSAs, the PFOS has the longest carbon chain with the most $\mathrm{F}$ atoms to form hydrogen bond, and the short chain PFSAs have much shorter carbon chains with fewer F atoms, leading to a relatively weaker adsorption, which is consistent with our experimental result in Support Information Text S4.

\subsection{DFT calculations of adsorption}

In this section we construct a system without water molecules to decrease the computational cost and to only consider the interaction between the hydrated hematite $\left(\begin{array}{llll}0 & 0 & 0 & 1\end{array}\right)$ surface and PFSAs. The computational parameters were set to lower accuracy to perform the calculations in suitable time. The modified parameters are as follows: the integration over the Brillouin zone was performed with a $1 \times 1 \times 1 \mathrm{k}$ point mesh generated following the Monkhorst-Pack scheme. The calculations were performed within the generalized gradient approximation (GGA) proposed by Perdew, Burke and Ernerhof (PBE). In the calculation of hematite surface and PFSAs, other changed details were electronic energy SCF tolerance, $1.0 \times 10^{-6} \mathrm{eV} /$ atom; energy change per ion, $\mathrm{dE} / \mathrm{ion}$, $1.0 \times 10^{-5} \mathrm{eV} /$ atom; maximum force, $0.03 \mathrm{eV} / \AA \AA \AA$; and maximum displacement, $1.0 \times 10^{-3} \AA$.

Six different adsorption configurations are shown in Fig. 5. Three PFSAs including PFBS, PFHxS and PFOS were chosen for typical case studies. The configurations A, B, C were constructed by MD simulations of hematite surfaces and PFSAs without the water molecules. Configurations D, E, F were some of the stable adsorption configurations obtained from previous MD simulations by simply deleting the water molecules. We note that there are many other possible adsorption configurations of PFSAs on the hematite surfaces, and the presented two configurations of each PFSA only represent the typical possible adsorption behaviour of PFSAs. Moreover, under the ambient environment conditions water molecules do exist; nevertheless, to reduce the computational and time cost, and for the purpose of exploring the interaction between the hematite surface and PFSAs, the water molecules were ignored.

The adsorption energy $\left(E_{a d s}\right)$ is derived according to the 
Table 1

Calculated results of adsorption energy of PFSAs on hydrated hematite surface.

\begin{tabular}{|c|c|c|c|c|}
\hline Adsorption type ${ }^{a}$ & $E_{\text {adsorbate }}(\mathrm{eV})$ & $E_{\text {surface }}(\mathrm{eV})$ & $E_{\text {surface after adsorption }}(\mathrm{eV})$ & $E_{a d s}(\mathrm{eV})$ \\
\hline A & -8179.568 & -189436.254 & -197616.860 & -1.038 \\
\hline B & -11136.985 & -189436.254 & -200574.018 & -0.779 \\
\hline $\mathrm{C}$ & -14094.373 & -189436.254 & -203531.394 & -0.767 \\
\hline $\mathrm{D}$ & -8179.568 & -189436.254 & -197616.315 & -0.493 \\
\hline $\mathrm{E}$ & -11136.985 & -189436.254 & -200573.815 & -0.576 \\
\hline $\mathrm{F}$ & -14094.373 & -189436.254 & -203530.826 & -0.199 \\
\hline
\end{tabular}

a Adsorption types are shown in Fig. 5.
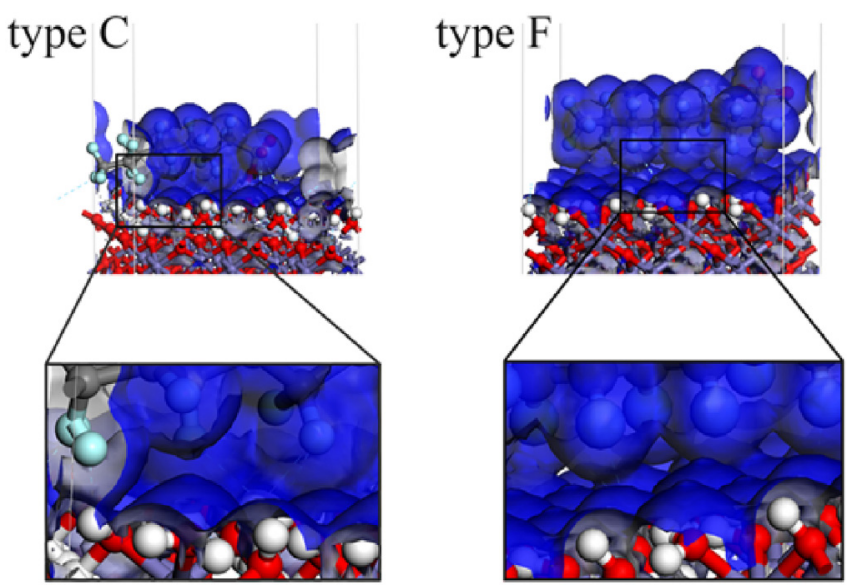

Fig. 6. Electron density isosurface of PFOS on hydrated hematite $\left(\begin{array}{llll}0 & 0 & 0 & 1\end{array}\right)$ surface with an isovalue of $0.05 \mathrm{e} / \AA^{3}$. To show clearly, partial enlarged views are exhibited.

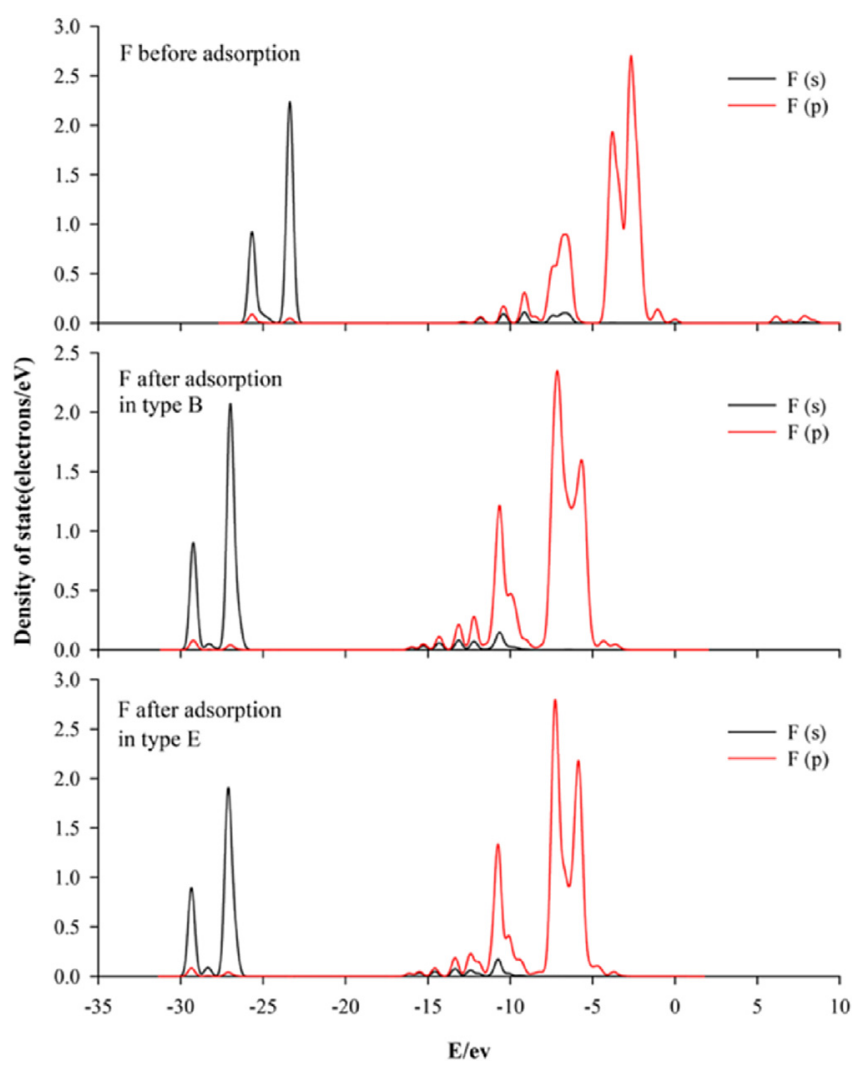

Fig. 7. PDOS of $\mathrm{F}$ atom on PFHxS tail before and after adsorption. Types B and E are shown in Fig. 5.

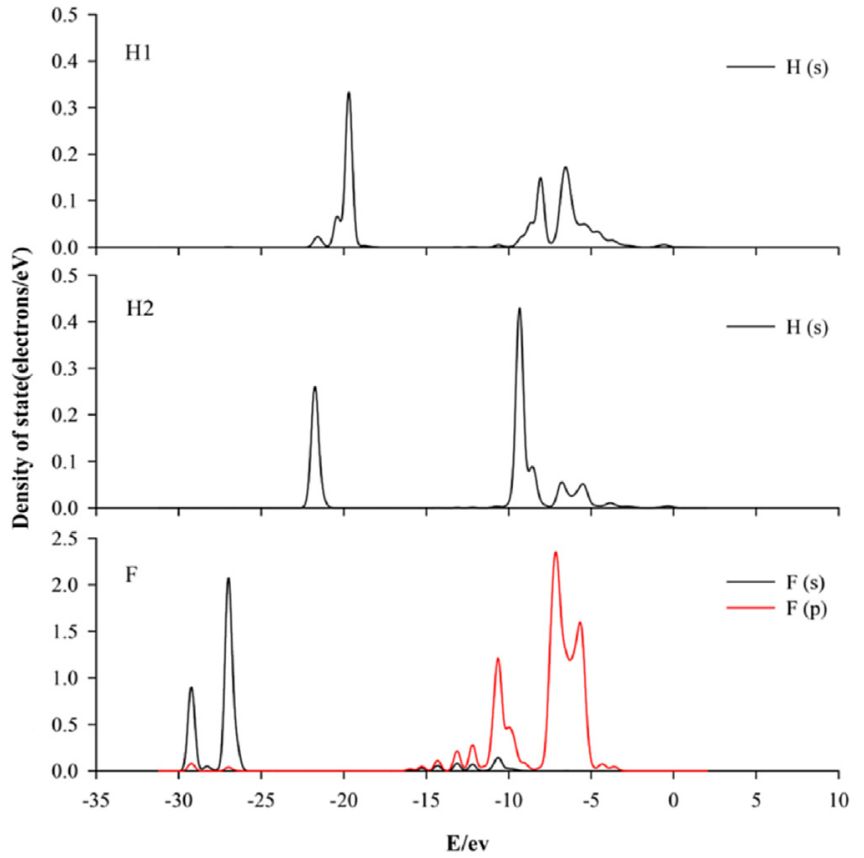

Fig. 8. PDOS of $\mathrm{F}$ atom on PFHxS tail and the adjacent $\mathrm{H}$ atoms on the surface. $\mathrm{H} 1$ and $\mathrm{H} 2$ are different hydrogen atoms.

following equation:

$E_{\text {ads }}=E_{\text {surface after adsorption }}-\left(E_{\text {surface }}+E_{\text {adsorbate }}\right)$

where $E_{\text {surface after adsorption, }} E_{\text {surface, }}$ and $E_{\text {adsorbate }}$ represent the total energy of the system, the energy of the hydrated hematite surface, and the energy of the PFSAs, respectively. The calculated energy of each contribution is listed in Table 1 . When PFSAs adsorb at the surface without the water molecules, the adsorption energy is negative in both adsorption models, meaning that the adsorption of PFSAs on hydrated hematite reduces the total energy. This result indicates that the PFSAs are able to lie on the surface, and the hydrogen bonds between $\mathrm{F}$ and $\mathrm{H}$ can lower the system energy. Furthermore, the different adsorption models show a trend that the $E_{\text {ads }}$ of $A, B, C$ are all lower than those of D, E, F, respectively; this may be the result of different adsorption geometries. We note that these configurations may not be the global minimum so it is not appropriate to use these results to assess the best structure for adsorption in vacuum. In general, both models will lead to a drop in the total energy. And certainly, the adsorption model with the long axis of PFSAs parallel to the surface can occur. Moreover, in aqueous conditions, fluorine atoms interact with surrounding water molecules as well as the hydrated surface. We roughly calculated the interaction energy between two water molecules and the interaction energy between a water molecule and a PFBS molecule. We 
found the optimized distance between the hydrogen atom in the water molecule to the oxygen atom in the other water molecule was approximately $2 \AA$, and the reduction in the energy was 0.21 $\mathrm{eV}-0.27 \mathrm{eV}$. The optimized distance between the hydrogen atom in the water molecule to the F atom on PFSAs was in the $2.5 \AA-2.8 \AA$ range, and the reduction in the energy was $0.009 \mathrm{eV}-0.014 \mathrm{eV}$, an order of magnitude lower than that the interaction between two water molecules. Therefore, the water molecules would tend to aggregate and exclude the PFSAs. Moreover, the adsorption energy of PFSAs on hematite overwhelmed the interaction between PFSAs and water molecules.

The electron density configuration gives us an intuitive picture of the PFASs adsorption on the hematite surface. Fig. 6 shows the three-dimensional isosurface plot of PFOS on the surface as an example with the isovalue of $0.05 \mathrm{e} / \AA^{3}$. The electron cloud of the $\mathrm{F}$ atom overlaps parts of the $\mathrm{H}$ atom on the hydrated hematite $(0$ 001 ) surface, indicating that $\mathrm{F}$ and $\mathrm{H}$ atoms play an important role during the adsorption process explicitly, also indicating that the hydrogen bond interaction participates in the adsorption.

Analysis of the electron structure based on the density of states (DOS) elucidates the nature of the bonds between the adsorbed PFASs and the hydrated hematite $\left(\begin{array}{llll}0 & 0 & 0 & 1\end{array}\right)$ surface. Fig. 7 shows the partial density of states (PDOS) of the F atom on the tail of PFHxS as an example. Comparing the $\mathrm{F}$ atom before and after the adsorption, it can be learned from the PDOS that in both adsorption type B and type $\mathrm{E}$, all the electronic density of states are lowered, with the peaks appearing between $-15 \mathrm{eV}$ and $-5 \mathrm{eV}$ and $-30 \mathrm{eV}$ to $-26 \mathrm{eV}$. This indicates the energies of the adsorbed $\mathrm{F}$ atoms are considerably lowered. Fig. 8 shows the PDOS of $F$ atom on PFHxS tail in adsorption type $\mathrm{B}$ and the adjacent $\mathrm{H}$ atoms on the surface. The $\mathrm{p}$ orbital of $\mathrm{F}$ atom is mainly localized at $-11 \mathrm{eV}$ to $-9 \mathrm{eV}$ and $-8 \mathrm{eV}$ to $-5 \mathrm{eV}$, where the $\mathrm{s}$ orbital of $\mathrm{H}$ atoms also have distribution. The overlap of the PDOS is one piece of evidence for bond formation between atom pairs (Guo et al., 2011), and hydrogen bonds formed by the overlap of $\mathrm{F}(p)$ orbital and $\mathrm{H}(s)$ orbital are sorbent-sorbate interactions which reveal the mechanism of PFSAs adsorbed on hydrated hematite surface.

\subsection{Discussion}

In the present study, we simulated a situation that was similar to the real environment to infer the PFSAs adsorption behaviour. The PFSA's adsorption model showed that the long axes lay along the surface, which is dissimilar to the adsorption geometries of alkyl sulfonic acid (e.g., sodium dodecyl sulfate SDS) and laurate on hematite surface (Chernyshova et al., 2011; Gao and Chorover, 2010). Johnson et al. (2007) speculated that the PFOS adsorbs onto Ottawa sand with the long axis parallel to the surface for the monolayer coverage; this is identical to as our model. Considering the size of PFOS and assuming that there is no space between the molecules, a density of $4 \mathrm{PFOS} / \mathrm{nm}^{2}$ should be obtained on the surface. In our simulation system, the density is only $0.16 \mathrm{PFOS} / \mathrm{nm}^{2}$ on the hematite surface, far below the coverage, and the adsorption model is consistent with the previous experimental study.

In recent years, a few studies reported that the short chain PFSAs are more mobile than PFOS in the environment. Based on the organic carbon normalized distribution coefficients $\left(\mathrm{K}_{\mathrm{OC}}\right)$, Zhou et al. (2013) speculated that the short chain PFAAs have lower adsorption potentials than PFOS and PFOA. Vierke et al. (2014) investigated the transport of PFAAs using a water-saturated sediment column and found higher mobilities for shorter chain PFAAs and an increasing trend of partition coefficients with increasing chain length. The analysis of our MD simulations was in good agreement with these experimental results.

The negative adsorption energies remind us that the configurations are energy permitted, but the obtained values need to be compared carefully for the adsorption configuration and consideration of different conditions. We also observed that the interface between water and the solid phase is complicated. In addition to hematite, the solid phase such as sediment and sludge plays an important role in the transport and fate of organic pollutants, and the mechanism needs to be investigated further.

\section{Conclusions}

In the present study, we have used computational methods to construct a system based on a hydrated hematite $\left(\begin{array}{llll}0 & 0 & 0 & 1\end{array}\right)$ surface as a model system to investigate the adsorption of PFSAs that occurred in environmental conditions. The molecular dynamic simulations showed that all PFSAs share the same adsorption configuration as the long carbon chains parallel to the surface. The formation of hydrogen bonds between $\mathrm{F}$ atom on PFSAs and $\mathrm{H}$ atoms on hydrated hematite surface helps to stabilize the unique configuration. Nevertheless, the water molecules trend to form hydrogen bonds between themselves. As a result, the sorption capacity increases with the increasing C-F chain length. Moreover, the adsorption energy and partial density of states (PDOS) analysis demonstrate the adsorption mechanism in between physical and chemical adsorption because the hydrogen bonds formed by the overlap of $\mathrm{F}$ $(p)$ orbital and $\mathrm{H}(s)$ orbital are weak intermolecular interactions while the physical adsorption are mainly ascribed to the electrostatic interactions.

Nowadays, these short chain PFSAs have given significant contributions to the total PFSAs in environment. The data of human and biota exposure to these chemicals are still limited, and considering the persistent characteristic accompanies with the transfer ability of short chain PFSAs, it is therefore irresponsible to release them into the environment today, and potential problems of short chain PFSAs should be brought to the forefront.

\section{Acknowledgements}

This work was jointly supported by the Chinese Academy of Sciences [grant number XDB14030500]; and the National Natural Science Foundation [grant numbers 21477154, 21277164, and 21177146].

\section{Appendix A. Supplementary data}

Supplementary data related to this article can be found at http:// dx.doi.org/10.1016/j.watres.2017.02.024.

\section{References}

Al-Abadleh, H.A., Grassian, V.H., 2003. Oxide surfaces as environmental interfaces. Surf. Sci. Rep. 52 (3-4), 63-161.

Alvarez-RamıRez, F., MartıNez-Magadán, J.M., Gomes, J.R.B., Illas, F., 2004. On the geometric structure of the (0001) hematite surface. Surf. Sci. 558 (1-3), 4-14.

Blake, R.L., Hessevick, R.E., Zoltai, T., Finger, L.W., 1966. Refinement of the hematite structure. Am. Mineral. 51, 123-129.

Brooke, D., Footitt, A., Nwaogu, T.A., 2004. Environmental Risk Evaluation Report: Perfluorooctane Sulfonate (PFOS). UK Environment Agency.

Catalano, J.G., 2011. Weak interfacial water ordering on isostructural hematite and corundum (001). Surfaces. Geochim. Cosmochim. Ac. 75 (8), 2062-2071.

Chambers, S.A., Yi, S.I., 1999. Fe termination for $\alpha-\mathrm{Fe}_{2} \mathrm{O}_{3}$ (0001) as grown by oxygenplasma-assisted molecular beam epitaxy. Surf. Sci. 439 (1), L785-L791.

Chernyshova, I.V., Ponnurangam, S., Somasundaran, P., 2011. Adsorption of fatty acids on iron (Hydr)Oxides from aqueous solutions. Langmuir 27 (16) 10007-10018.

Clark, S.J., Segall, M.D., Pickard, C.J., Hasnip, P.J., Probert, M.I.J., Refson, K., Payne, M.C., 2005. First principles methods using castep. Z. Krist. 220 (5/6) 2005), 567-570.

Eggleston, C.M., Stack, A.G., Rosso, K.M., Higgins, S.R., Bice, A.M., Boese, S.W. Pribyl, R.D., Nichols, J.J., 2003. The structure of hematite $\left(\alpha-\mathrm{Fe}_{2} \mathrm{O}_{3}\right)(001)$ surfaces in aqueous media: scanning tunneling microscopy and resonant tunneling 
calculations of coexisting $\mathrm{O}$ and Fe terminations. Geochim. Cosmochim. Ac 67 (5), 985-1000.

Finger, L.W., Hazen, R.M., 1980. Crystal structure and isothermal compression of $\mathrm{Fe}_{2} \mathrm{O}_{3}, \mathrm{Cr}_{2} \mathrm{O}_{3}$, and $\mathrm{V}_{2} \mathrm{O}_{3}$ to 50 kbars. J. Appl. Phys. 51 (10), 5362-5367.

Gao, X., Chorover, J., 2010. Adsorption of sodium dodecyl sulfate (SDS) at ZnSe and Alpha- $\mathrm{Fe}_{2} \mathrm{O}_{3}$ surfaces: combining infrared spectroscopy and batch uptake studies. J. Colloid Interf. Sci. 348 (1), 167-176.

Gao, X., Chorover, J., 2012. Adsorption of perfluorooctanoic acid and perfluorooctanesulfonic acid to iron oxide surfaces as studied by flow-through atrftir spectroscopy. Environ. Chem. 9 (2), 148-157.

Giesy, J.P., Kannan, K., 2001. Global distribution of perfluorooctane sulfonate in wildlife. Environ. Sci. Technol. 35 (7), 1339-1342.

Guo, Y.-n., Lu, X., Zhang, H.-p., Weng, J., Watari, F., Leng, Y., 2011. DFT study of the adsorption of aspartic acid on pure, N-Doped, and Ca-Doped rutile (110). Surfaces. J. Phys. Chem. C 115 (38), 18572-18581.

Hohenberg, P., Kohn, W., 2010. Inhomogeneous electron gas. Phys. Rev. 136 (3), $864-871$.

Inc, A.S, 2012. Materials Studio Release Notes, Release 6.1. Accelrys Software Inc, San Diego.

Johnson, R.L., Anschutz, A.J., Smolen, J.M., Simcik, M.F., Penn, R.L., 2007. The adsorption of perfluorooctane sulfonate onto sand, clay, and iron oxide surfaces. J. Chem. Eng. Data 52 (4), 1165-1170.

Jr, B.G., Foster, A.L., Ostergren, J.D., 1999a. Mineral surfaces and bioavailability of heavy metals: a molecular-scale perspective. Proc. Natl. Acad. Sci. U. S. A. 96 (7), 3388-3395.

Jr, B.G., Henrich, V.E., Casey, W.H., Clark, D.L., Eggleston, C., Felmy, A., Goodman, D.W., Grätzel, M., Maciel, G., Mccarthy, M.I., 1999b. Metal oxide surfaces and their interactions with aqueous solutions and microbial organisms. Chem. Rev. 99 (1), 77-174.

Kannan, K., Corsolini, S., Falandysz, J., Fillmann, G., Kumar, K.S., Loganathan, B.G., Mohd, M.A., Olivero, J., Van, W.N., Yang, J.H., 2004. Perfluorooctanesulfonate and related fluorochemicals in human blood from several countries. Environ. Sci. Technol. 38 (17), 4489-4495.

Karasawa, N., Iii, W.A.G., 1989. Acceleration of convergence for lattice sums. J. Phys. Chem. 93 (21), 7320-7327.

Kerisit, S., 2011. Water structure at hematite-water interfaces. Geochim. Cosmochim. Ac 75 (8), 2043-2061.

Kohn, W., Sham, L.J., 1965. Self-consistent equations including exchange and correlation effects. Phys. Rev. 140 (4A), 1133-1138.

Lange, F.T., Wenz, M., Schmidt, C.K., Brauch, H.J., 2007. Occurrence of perfluoroalkyl sulfonates and carboxylates in German drinking water sources compared to other countries. Water Sci. Technol. 56 (11), 151-158.

Li, C., Monti, S., Carravetta, V., 2012. Journey toward the surface: how Glycine adsorbs on titania in water solution. J. Phys. Chem. C 116 (34), 18318-18326.

Liu, P.N., Kendelewicz, T., Brown, G.E., Nelson, E.J., Chambers, S.A., 1998. Reaction of water vapor with Alpha- $\mathrm{Al}_{2} \mathrm{O}_{3}$ and Alpha- $\mathrm{Fe}_{2} \mathrm{O}_{3}$ (0001) surfaces: synchrotron Xray photoemission studies and thermodynamic calculations. Surf. Sci. 417.

Lu, Y., Wang, Y., Zhu, W., 2010. Nonbonding interactions of organic halogens in biological systems: implications for drug discovery and biomolecular design. Phys. Chem. Chem. Phys. 12 (18), 4543-4551.

Martin, G.J., Cutting, R.S., Vaughan, D.J., Warren, M.C., 2009. Bulk and key surface structures of hematite, magnetite, and goethite: a density functional theory study. Am. Mineral. 94 (10), 1341-1350.

Mason, S.E., Iceman, C.R., Tanwar, K.S., Trainor, T.P., Chaka, A.M., 2009. Pb(II) adsorption on isostructural hydrated alumina and hematite (0001) surfaces: a DFT study. J. Phys. Chem. C 113 (6), 2159-2170.

Matyszewska, Dorota, Tappura, Kirsi, Greger Orädd, A., Bilewicz, Renata, 2007. Influence of perfluorinated compounds on the properties of model lipid membranes. J. Phys. Chem. B 111 (33), 9908-9918.

Moller, A., Ahrens, L., Surm, R., Westerveld, J., van der Wielen, F., Ebinghaus, R., de Voogt, P., 2010. Distribution and sources of polyfluoroalkyl substances (PFAS) in the river rhine watershed. Environ. Pollut. 158 (10), 3243-3250.

Nosé, S., 1984. A unified formulation of the constant temperature molecular dynamics methods. J. Phys. Chem. 6 (2), 25-31.
Nosé, S., 1991. Constant temperature molecular dynamics methods. Prog. Theor. Phys. Supp 103 (103), 1-46.

Nosé, S., 2006. A molecular dynamics method for simulations in the canonical ensemble. Mol. Phys. 52 (2), 255-268.

Notman, R., Walsh, T.R., 2009. Molecular dynamics studies of the interactions of water and amino acid analogues with quartz surfaces. Langmuir 25 (3) $1638-1644$.

Olsen, G.W., Chang, S.C., Noker, P.E., Gorman, G.S., Ehresman, D.J., Lieder, P.H., Butenhoff, J.L., 2009. A comparison of the pharmacokinetics of perfluorobutanesulfonate (PFBS) in rats, monkeys, and humans. Toxicology 256 $(1-2), 65-74$.

Rahman, M.F., Peldszus, S., Anderson, W.B., 2014. Behaviour and fate of perfluoroalkyl and polyfluoroalkyl substances (PFSAs) in drinking water treatment: a review. Water Res. 50, 318-340.

Rollmann, G., Rohrbach, A., Entel, P., Hafner, J., 2004. First-principles calculation of the structure and magnetic phases of hematite. Phys. Rev. B 69 (16), 1124-1133.

Souvi, S.M.O., Badawi, M., Paul, J.-F., Cristol, S., Cantrel, L., 2013. A DFT study of the hematite surface state in the presence of $\mathrm{H}_{2}, \mathrm{H}_{2} \mathrm{O}$ and $\mathrm{O}_{2}$. Surf. Sci. 610, 7-15.

Steinle-Darling, E., Reinhard, M., 2008. Nanofiltration for trace organic contaminant removal: structure, solution, and membrane fouling effects on the rejection of perfluorochemicals. Environ. Sci. Technol. 42 (14), 5292-5297.

Sun, H., 1998. Compass: an ab initio force-field optimized for condensed-phase applicationsoverview with details on alkane and benzene compounds. J. Phys. Chem. B 102 (38), 7338-7364.

Sun, H., Ren, P., Fried, J.R., 1998. The compass force field: Param. Validation Phosphazenes. Comput. Theor. Polym. Sci. 8 (1-2), 229-246.

Tanwar, K.S., Lo, C.S., Eng, P.J., Catalano, J.G., Walko, D.A., Brown, G.E., Waychunas, G.A., Chaka, A.M., Trainor, T.P., Fairbanks, U.O.A., 2007. Surface diffraction study of the hydrated hematite (1 110 2). Surf. Surf. Sci. 601 (2) 460-474.

Thomas, N.J., 2006. Association between perfluorinated compounds and pathological conditions in southern sea otters. Environ. Sci. Technol. 40 (16), 4943-4948.

Trainor, T.P., Chaka, A.M., Eng, P.J., Newville, M., Waychunas, G.A., Catalano, J.G., Brown, G.E., 2004. Structure and reactivity of the hydrated hematite (0001). Surf. Surf. Sci. 573 (2), 204-224.

UNEP, 2009. The nine new pops. In: An Introduction to the Nine Chemicals Added to the Stockholm Convention by the Conference of the Parties at its Fourth Meeting. Available online: http://chm.pops.int/Programmes/NewPOPs/ Publications/tabid/695/language/en-US/D-efault.aspx.

Vierke, L., Berger, U., Cousins, IT., 2013. Estimation of the acid dissociation constant of perfluoroalkyl carboxylic acids through an experimental investigation of their water-to-air transport. Environ. Sci. Technol. 47 (19), 11032-11039.

Vierke, L., Moller, A., Klitzke, S., 2014. Transport of perfluoroalkyl acids in a watersaturated sediment column investigated under near-natural conditions. Environ. Pollut. 186, 7-13.

Wang, X.G., Weiss, W., Shaikhutdinov, S.K., Ritter, M., Petersen, M., Wagner, F., Schl, Ouml, R., Scheffler, M., 1998. The hematite $\left(\alpha-\mathrm{Fe}_{2} \mathrm{O}_{3}\right)(0001)$ surface: evidence for domains of distinct chemistry. Phys. Rev. Lett. 81 (5), 1038-1041.

Wang, Z., Cousins, I.T., Scheringer, M., Hungerbühler, K., 2013. Fluorinated alternatives to long-chain perfluoroalkyl carboxylic acids (PFCAs), perfluoroalkane sulfonic acids (PFSAs) and their potential precursors. Environ. Int. 60, 242-248.

Wasserman, E., Rustad, J.R., Felmy, A.R., Hay, B.P., Halley, J.W., 1997. Ewald methods for polarizable surfaces with application to hydroxylation and hydrogen bonding on the (012) and (001) surfaces of $\alpha-\mathrm{Fe}_{2} \mathrm{O}_{3}$. Surf. Sci. 385 (2-3), 217-239.

Williams, D.E., 2007. Accelerated convergence of crystal-lattice potential sums. Acta Crystallogr. A 27 (5), 452-455.

Zhao, L., Zhang, Y., Fang, S., Zhu, L., Liu, Z., 2014. Comparative sorption and desorption behaviors of PFHxS and PFOS on sequentially extracted humic substances. J. Environ. Sci. 26 (12), 2517-2525.

Zhou, Z., Liang, Y., Shi, Y., Xu, L., Cai, Y., 2013. Occurrence and transport of perfluoroalkyl acids (PFAAs), including short-chain PFAAs in Tangxun Lake, China. Environ. Sci. Technol. 47 (16), 9249-9257. 